\title{
NONLOCAL SOLVABILITY CONDITIONS FOR CAUCHY PROBLEM FOR A SYSTEM OF FIRST ORDER PARTIAL DIFFERENTIAL EQUATIONS WITH SPECIAL RIGHT-HAND SIDES
}

\author{
M.V. DONTSOVA
}

\begin{abstract}
We consider a Cauchy problem for a system of two quasilinear first order partial differential equations with special right-hand sides. We obtain the conditions of a nonlocal solvability of this Cauchy problem. The study of the nonlocal solvability of the Cauchy problem for a system of two quasilinear differential equations with special right-hand sides is based on the method of an additional argument. The proof of the nonlocal solvability of the Cauchy problem for a system of two quasilinear first order partial differential equations with special right-hand sides relies on global estimates.
\end{abstract}

Keywords: first order partial differential equations, Cauchy problem, the method of an additional argument.

Mathematics Subject Classification: 35F50, 35F55, 35A01, 35A02, 35A05

\section{INTRODUCTION}

There are various methods for studying solvability of systems of first order partial differential equations. For instance, there are classical methods of characteristics, Galerkin's method, flows methods, additional argument method [1].

The additional argument method is a new way for studying solvability of systems of first order partial differential equations. It does not replace other known methods but complements them [2]. In many cases the application of this method allows one to find more effectively and specifically the conditions of local solvability in original coordinates for systems of nonlinear and quasi-linear first order differential equations without employing inverse function theorem [2]-[13].

In work 3] first by means of the additional argument method there were determined the nonlocal solvability conditions for the Cauchy problem for the systems

$$
\left\{\begin{array}{l}
\partial_{t} u(t, x)+(a u(t, x)+b v(t, x)) \partial_{x} u(t, x)=0, \\
\partial_{t} v(t, x)+(c u(t, x)+g v(t, x)) \partial_{x} v(t, x)=0,
\end{array}\right.
$$

where $u(t, x), v(t, x)$ are unknown functions, $a, c, b, g$ are known positive constants, $(t, x) \in \Omega_{T}$, where $\Omega_{T}=\{(t, x) \mid 0 \leqslant t \leqslant T, x \in(-\infty, \infty), T>0\}$ subject to the initial conditions

$$
u(0, x)=\varphi_{1}(x), \quad v(0, x)=\varphi_{2}(x),
$$

$\varphi_{1}(x), \varphi_{2}(x)$ are given functions.

M.V. Dontsova, Nonlocal solvability CONDitions for CAuChy PRoblem for a System OF FIRST ORDER PARTIAL DIFFERENTIAL EQUATIONS WITH SPECIAL RIGHT-HAND SIDES.

(C) Dontsova M.V. 2014.

Submitted June 30, 2014. 
By means of the additional argument method in work [4] there were found nonlocal solvability conditions for the Cauchy problem for the systems

$$
\left\{\begin{array}{l}
\partial_{t} u(t, x)+\left(a u(t, x)+b v(t, x)+h_{1}\right) \partial_{x} u(t, x)=f_{1}(t, x), \\
\partial_{t} v(t, x)+\left(c u(t, x)+g v(t, x)+h_{2}\right) \partial_{x} v(t, x)=f_{2}(t, x),
\end{array}\right.
$$

subject to initial condition (2), where $u(t, x), v(t, x)$ are unknown functions, $a, c, b, g, h_{1}, h_{2}$ are known positive constants, $f_{1}, f_{2}$ are given functions, $(t, x) \in \Omega_{T}$.

Systems (1), (3) appear in various problems in natural sciences, for instance, in describing the spreading of finite intensity perturbation under non-stationary one-dimensional flow of ideal gas [5]. A particular example of such systems is the Frankl system of equations [6], [7].

We consider the system

$$
\left\{\begin{array}{l}
\partial_{t} u(t, x)+\left(a_{1} u(t, x)+b_{1} v(t, x)\right) \partial_{x} u(t, x)=a_{2} u(t, x)+b_{2} v(t, x), \\
\partial_{t} v(t, x)+\left(c_{1} u(t, x)+g_{1} v(t, x)\right) \partial_{x} v(t, x)=g_{2} v(t, x),
\end{array}\right.
$$

where $u(t, x), v(t, x)$ are unknown functions, $a_{1}, b_{i}, c_{1}, g_{1}, i=1,2$, are given positive constants, $a_{2}, g_{2}$ are given constants.

Various methods were applied for studying the solvability of a system close to (4). The description of many modern approaches is contained [1]. For instance, in [1] there was made an analysis of solvability on the basis of the classical methods of characteristics as well as by employing the notion of the generalized solution. Both these approaches, as many others, have their advantages and disadvantages. For instance, in the method of characteristics the solvability condition in the original coordinates is the existence of the inverse function for the solution to a characteristic equation. Finding the inverse function is a more complicated problem than the original one. This is one usually does not solve it and assume instead the possibility of the inverse change of variables [1].

In the present work by means of the additional argument method we determine the nonlocal solvability conditions for the Cauchy problem for system (4) subject to initial conditions (2) on $\Omega_{T}$, where $a_{1}, b_{i}, c_{1}, g_{1}, i=1,2$, are given positive constants, $a_{2}, g_{2}$ are given constants.

In accordance with the additional argument method, for problem (4), (2) we write the extended characteristic system [7]-[11]:

$$
\begin{aligned}
& \frac{d \eta_{1}(s, t, x)}{d s}=a_{1} u\left(s, \eta_{1}(s, t, x)\right)+b_{1} v\left(s, \eta_{1}(s, t, x)\right), \\
& \frac{d \eta_{2}(s, t, x)}{d s}=c_{1} u\left(s, \eta_{2}(s, t, x)\right)+g_{1} v\left(s, \eta_{2}(s, t, x)\right), \\
& \frac{d u\left(s, \eta_{1}(s, t, x)\right)}{d s}=a_{2} u\left(s, \eta_{1}(s, t, x)\right)+b_{2} v\left(s, \eta_{1}(s, t, x)\right), \\
& \frac{v\left(s, \eta_{2}(s, t, x)\right)}{d s}=g_{2} v\left(s, \eta_{2}(s, t, x)\right), \\
& \eta_{1}(t, t, x)=x, \eta_{2}(t, t, x)=x, \\
& u\left(0, \eta_{1}(0, t, x)\right)=\varphi_{1}\left(\eta_{1}(0, t, x)\right), v\left(0, \eta_{2}(0, t, x)\right)=\varphi_{2}\left(\eta_{2}(0, t, x)\right) .
\end{aligned}
$$

The feature of this system is that it involves unknown functions as superpositions that complicates essentially the proof of solvability.

We introduce new unknown functions:

$$
\begin{array}{ll}
w_{1}(s, t, x)=u\left(s, \eta_{1}(s, t, x)\right), & w_{2}(s, t, x)=v\left(s, \eta_{2}(s, t, x)\right), \\
w_{3}(s, t, x)=v\left(s, \eta_{1}(s, t, x)\right), & w_{4}(s, t, x)=u\left(s, \eta_{2}(s, t, x)\right) .
\end{array}
$$

Then the extended characteristic system casts into the form:

$$
\frac{d \eta_{1}(s, t, x)}{d s}=a_{1} w_{1}(s, t, x)+b_{1} w_{3}(s, t, x)
$$




$$
\begin{aligned}
& \frac{d \eta_{2}(s, t, x)}{d s}=c_{1} w_{4}(s, t, x)+g_{1} w_{2}(s, t, x) \\
& \frac{d w_{1}(s, t, x)}{d s}=a_{2} w_{1}(s, t, x)+b_{2} w_{3}(s, t, x) \\
& \frac{d w_{2}(s, t, x)}{d s}=g_{2} w_{2}(s, t, x) \\
& w_{3}(s, t, x)=w_{2}\left(s, s, \eta_{1}\right), w_{4}(s, t, x)=w_{1}\left(s, s, \eta_{2}\right) \\
& \eta_{1}(t, t, x)=x, \eta_{2}(t, t, x)=x \\
& w_{1}(0, t, x)=\varphi_{1}\left(\eta_{1}(0, t, x)\right), w_{2}(0, t, x)=\varphi_{2}\left(\eta_{2}(0, t, x)\right)
\end{aligned}
$$

Unknown functions $\eta_{i}, w_{j}, i=1,2, j=\overline{1,4}$ depend not only on $t$ and $x$, but also on additional argument $s$. Integrating equations (11)-(14) w.r.t. argument $s$ and taking into considerations conditions (15)-(17), we obtain the equivalent system of integral equations:

$$
\begin{aligned}
& \eta_{1}(s, t, x)=x-\int_{s}^{t}\left(a_{1} w_{1}+b_{1} w_{3}\right) d \tau, \\
& \eta_{2}(s, t, x)=x-\int_{s}^{t}\left(c_{1} w_{4}+g_{1} w_{2}\right) d \tau, \\
& w_{1}(s, t, x)=\varphi_{1}\left(\eta_{1}(0, t, x)\right)+\int_{0}^{s}\left(a_{2} w_{1}+b_{2} w_{3}\right) d \tau, \\
& w_{2}(s, t, x)=\varphi_{2}\left(\eta_{2}(0, t, x)\right)+\int_{0}^{s} g_{2} w_{2} d \tau, \\
& w_{3}(s, t, x)=w_{2}\left(s, s, \eta_{1}\right), \\
& w_{4}(s, t, x)=w_{1}\left(s, s, \eta_{2}\right) .
\end{aligned}
$$

Substituting (18), (19) into (20)-(23), we get

$$
\begin{aligned}
& w_{1}(s, t, x)=\varphi_{1}\left(x-\int_{0}^{t}\left(a_{1} w_{1}+b_{1} w_{3}\right) d \tau\right)+\int_{0}^{s}\left(a_{2} w_{1}(\tau, t, x)+b_{2} w_{3}(\tau, t, x)\right) d \tau, \\
& w_{2}(s, t, x)=\varphi_{2}\left(x-\int_{0}^{t}\left(c_{1} w_{4}(\tau, t, x)+g_{1} w_{2}(\tau, t, x)\right) d \tau\right)+\int_{0}^{s} g_{2} w_{2}(\tau, t, x) d \tau, \\
& w_{3}(s, t, x)=w_{2}\left(s, s, x-\int_{s}^{t}\left(a_{1} w_{1}+b_{1} w_{3}\right) d \tau\right), \\
& w_{4}(s, t, x)=w_{1}\left(s, s, x-\int_{s}^{t}\left(c_{1} w_{4}+g_{1} w_{2}\right) d \tau\right) .
\end{aligned}
$$

We shall write that constants $K_{0}, K_{1}, K_{2}, \ldots$ are determined by the initial data if these constants are determined by given characteristics of the problem, the norms and the extrema of known functions by means of closed algebraic, differential or integral expressions, i.e., in the framework of the original problem they can be expressed by a particular number.

The following lemma holds true. 
Lemma 1. Suppose that functions $w_{1}(s, t, x), w_{2}(s, t, x)$ solve system of integral equations (24) -(27), are continuously differentiable and bounded together with its first derivatives. Then functions $u(t, x)=w_{1}(t, t, x), v(t, x)=w_{2}(t, t, x)$ solve problem (4), (2) on $\Omega_{T_{0}}, T_{0} \leqslant T$, where $T_{0}$ is a constant determined by the given data.

Lemma 1 is the basis of the additional argument. Lemma 1 can be proven in the same way as in works [7]-[11].

\section{EXISTENCE OF LOCAL SOLUTION}

In order to prove the solvability of problem (4), (2) in the class of bounded functions, we shall make use system of integral equations (24)-(27).

We denote

$$
\begin{aligned}
& \Gamma_{T}=\{(s, t, x) \mid 0 \leqslant s \leqslant t \leqslant T, x \in(-\infty,+\infty), T>0\}, \\
& C_{\varphi}=\max \left\{\sup _{\mathbb{R}} \mid \varphi_{i}^{(l)} \| i=1,2, l=\overline{0,2}\right\}, \quad l=\max \left\{a_{1},\left|a_{2}\right|, b_{1}, b_{2}, c_{1}, g_{1},\left|g_{2}\right|\right\}, \\
& \|U\|=\sup _{\Gamma_{T}}|U(s, t, x)|, \quad\|f\|=\sup _{\Omega_{T}}|f(t, x)|,
\end{aligned}
$$

and $\bar{C}^{1,2,2}\left(\Omega_{T}\right)$ stands for the space of functions differentiable w.r.t. $t$, twice differentiable w.r.t. $x$, having mixed second order derivatives and bounded together with its derivatives on $\Omega_{T}, \bar{C}^{\alpha_{1}, \alpha_{2}, \ldots \alpha_{n}}\left(\Omega_{*}\right)$ indicates the space of functions continuous and bounded together with its derivatives up to order $\alpha_{m}$ w.r.t. $m$ th argument, $m=\overline{1, n}$ on unbounded subset $\Omega_{*} \subset \mathbb{R}^{n}$, $n=1,2, \ldots$

We introduce conditions playing the key role in the proof of nonlocal solvability of Cauchy problem (4), (2):

$$
a_{1}>0, \quad b_{i}>0, \quad c_{1}>0, \quad g_{1}>0, \quad \varphi_{1}^{\prime}(x) \geqslant 0, \quad \varphi_{2}^{\prime}(x) \geqslant 0, \quad i=1,2 .
$$

In the following theorem we formulate the conditions for the existence of a local solution to Cauchy problem (4), (2), whose smoothness w.r.t. $x$ is not less than for the initial data.

Theorem 1. Suppose that $\varphi_{i} \in \bar{C}^{2}(\mathbb{R}), i=1,2$, and conditions $(28)$ hold true. Then for each $T_{2}>0$, where $T_{2}=\min \left(\frac{1}{25 C_{\varphi} l}, \frac{1}{10 l}\right)$, Cauchy problem (4), (2) has the unique solution $u(t, x), v(t, x) \in \bar{C}^{1,2,2}\left(\Omega_{T_{2}}\right)$ determined by system of integral equations (24)-27).

We split the proof of this theorem into two lemmata.

Lemma 2. System of integral equations 24) -27) has the unique solution $w_{j} \in \bar{C}^{1,1,1}\left(\Gamma_{T_{2}}\right)$, where $j=\overline{1,4}, T_{2}=\min \left(\frac{1}{25 C_{\varphi} l}, \frac{1}{10 l}\right)$.

Proof. We prove this lemma by the scheme described in [13]. This is why we provide only key milestones.

We define the zero approximation of the solution to system of integral equations (24)-(27) by the identities:

$$
w_{10}(s, t, x)=\varphi_{1}(x), \quad w_{20}(s, t, x)=\varphi_{2}(x), \quad w_{30}(s, t, x)=\varphi_{2}(x), \quad w_{40}(s, t, x)=\varphi_{1}(x) .
$$

The first and subsequent approximations for system of equations (24)-(27) are introduced by means of the recurrent sequence of systems of equations $(n=1,2, \ldots)$ :

$$
w_{1 n}(s, t, x)=\varphi_{1}\left(x-\int_{0}^{t}\left(a_{1} w_{1 n}+b_{1} w_{3 n}\right) d \tau\right)+\int_{0}^{s}\left(a_{2} w_{1 n}+b_{2} w_{3 n}\right) d \tau
$$




$$
\begin{aligned}
& w_{2 n}(s, t, x)=\varphi_{2}\left(x-\int_{0}^{t}\left(c_{1} w_{4 n}(\tau, t, x)+g_{1} w_{2 n}(\tau, t, x)\right) d \tau\right)+\int_{0}^{s} g_{2} w_{2 n}(\tau, t, x) d \tau, \\
& w_{3 n}(s, t, x)=w_{2(n-1)}\left(s, s, x-\int_{s}^{t}\left(a_{1} w_{1 n}+b_{1} w_{3 n}\right) d \tau\right), \\
& w_{4 n}(s, t, x)=w_{1(n-1)}\left(s, s, x-\int_{s}^{t}\left(c_{1} w_{4 n}+g_{1} w_{2 n}\right) d \tau\right) .
\end{aligned}
$$

We define the zero approximation for system of equations $29-(32)$ by the identities $w_{j n}^{0}=$ $w_{j(n-1)}, j=\overline{1,4}$.

The first and subsequent approximations for system of equations $(29)-(32)$ are introduced by the formulae

$$
\begin{aligned}
& w_{1 n}^{k+1}(s, t, x)=\varphi_{1}\left(x-\int_{0}^{t}\left(a_{1} w_{1 n}^{k}+b_{1} w_{3 n}^{k}\right) d \tau\right)+\int_{0}^{s}\left(a_{2} w_{1 n}^{k}+b_{2} w_{3 n}^{k}\right) d \tau, \\
& w_{2 n}^{k+1}(s, t, x)=\varphi_{2}\left(x-\int_{0}^{t}\left(c_{1} w_{4 n}^{k}(\tau, t, x)+g_{1} w_{2 n}^{k}(\tau, t, x)\right) d \tau\right)+\int_{0}^{s} g_{2} w_{2 n}^{k}(\tau, t, x) d \tau, \\
& w_{3 n}^{k+1}(s, t, x)=w_{2(n-1)}\left(s, s, x-\int_{s}^{t}\left(a_{1} w_{1 n}^{k}+b_{1} w_{3 n}^{k}\right) d \tau\right) \\
& w_{4 n}^{k+1}(s, t, x)=w_{1(n-1)}\left(s, s, x-\int_{s}^{t}\left(c_{1} w_{4 n}^{k}+g_{1} w_{2 n}^{k}\right) d \tau\right) .
\end{aligned}
$$

In the same way as in [13] we prove that for each $0 \leqslant t \leqslant T_{1}$, where $T_{1}=\min \left(\frac{1}{20 C_{\varphi} l}, \frac{1}{4 l}\right)$, successive approximations $(33)-(36)$ are bounded, continuous and converge to a continuous solution to system (29)-(32) satisfying the estimates $\left\|w_{j n}\right\| \leqslant 2 C_{\varphi}, j=\overline{1,4}$.

In the same as in [13], we establish that for each $0 \leqslant t \leqslant T_{1}$, where $T_{1}=\min \left(\frac{1}{20 C_{\varphi} l}, \frac{1}{4 l}\right)$, there exist the derivatives $\partial_{x} w_{j n}, j=\overline{1,4}$ and the estimates

$$
\left\|\partial_{x} w_{1 n}\right\| \leqslant 4 C_{\varphi}, \quad\left\|\partial_{x} w_{2 n}\right\| \leqslant 4 C_{\varphi}, \quad\left\|\partial_{x} w_{3 n}\right\| \leqslant 6 C_{\varphi}, \quad\left\|\partial_{x} w_{4 n}\right\| \leqslant 6 C_{\varphi}
$$

hold true.

For each $0 \leqslant t \leqslant T_{1}$, where $T_{1}=\min \left(\frac{1}{20 C_{\varphi} l}, \frac{1}{4 l}\right)$, the successive approximations determined by system $(29)-(32)$ converge to the solution of system $24-(27)$ and the estimates

$$
\left\|w_{j}\right\| \leqslant 2 C_{\varphi}, \quad j=\overline{1,4}
$$

hold true. Then we prove that $w_{j n x} \rightarrow w_{j x}=\partial_{x} w_{j}, j=\overline{1,4}$, where functions $\partial_{x} w_{j}$ are continuous w.r.t. all its arguments on $\Gamma_{T_{2}}, T_{2}=\min \left(\frac{1}{25 C_{\varphi} l}, \frac{1}{10 l}\right)$. The estimates $\left\|\partial_{x} w_{i}\right\| \leqslant 4 C_{\varphi}$, $i=1,2,\left\|\partial_{x} w_{3}\right\| \leqslant 6 C_{\varphi},\left\|\partial_{x} w_{4}\right\| \leqslant 6 C_{\varphi}$ are valid.

In the same way we prove that $w_{j}, j=\overline{1,4}$ have continuous and bounded derivatives w.r.t. $t$ on $\Gamma_{T_{2}}$. The uniqueness of the solutions can be proven in the same as in paper [13].

Lemma 3. Under conditions (28) functions $\left\{w_{j}\right\}, j=\overline{1,4}$, being solutions to the system of equations (24) - 27), have continuous and bounded derivatives $\frac{\partial^{2} w_{j}}{\partial x^{2}}, \frac{\partial^{2} w_{j}}{\partial x t}, j=\overline{1,4}$, in $\Gamma_{T_{2}}$, where $T_{2}=\min \left(\frac{1}{25 C_{\varphi} l}, \frac{1}{10 l}\right)$. 
Proof. We differentiate twice successive approximations (29)-32 w.r.t. $x$ and denote $\omega_{j}^{n}=$ $w_{j n x x}, j=\overline{1,4}$. It yields the system of equations:

$$
\begin{aligned}
& \omega_{1}^{n}=-\varphi_{1}^{\prime} \int_{0}^{t}\left(a_{1} \omega_{1}^{n}+b_{1} \omega_{3}^{n}\right) d \tau+\int_{0}^{s}\left(a_{2} \omega_{1}^{n}+b_{2} \omega_{3}^{n}\right) d \tau \\
&+\varphi_{1}^{\prime \prime} \cdot\left(1-\int_{0}^{t}\left(a_{1} w_{1 n x}+b_{1} w_{3 n x}\right) d \tau\right)^{2}, \\
& \omega_{2}^{n}=-\varphi_{2}^{\prime} \int_{0}^{t}\left(c_{1} \omega_{4}^{n}+g_{1} \omega_{2}^{n}\right) d \tau+\int_{0}^{s} g_{2} \omega_{2}^{n} d \tau+\varphi_{2}^{\prime \prime} \cdot\left(1-\int_{0}^{t}\left(c_{1} w_{4 n x}+g_{1} w_{2 n x}\right) d \tau\right)^{2}, \\
& \omega_{3}^{n}=\omega_{2}^{n-1} \cdot\left(1-\int_{s}^{t}\left(a_{1} w_{1 n x}+b_{1} w_{3 n x}\right) d \tau\right)^{2}-w_{2(n-1) x} \int_{s}^{t}\left(a_{1} \omega_{1}^{n}+b_{1} \omega_{3}^{n}\right) d \tau, \\
& \omega_{4}^{n}=\omega_{1}^{n-1} \cdot\left(1-\int_{s}^{t}\left(c_{1} w_{4 n x}+g_{1} w_{2 n x}\right) d \tau\right)^{2}-w_{1(n-1) x} \int_{s}\left(c_{1} \omega_{4}^{n}+g_{1} \omega_{2}^{n}\right) d \tau .
\end{aligned}
$$

Under conditions (28), in view of the above established estimates $\left\|w_{j n}\right\| \leqslant 2 C_{\varphi}, j=\overline{1,4}$, we have $\left|\int_{s}^{t}\left(a w_{1 n}+b w_{3 n}\right) d \tau\right| \leqslant t l\left(\left\|w_{1 n}\right\|+\left\|w_{3 n}\right\|\right) \leqslant 4 t l C_{\varphi} \leqslant \frac{4 l C_{\varphi}}{25 l C_{\varphi}} \leqslant 0.16$. In the same way, $\left|\int_{s}^{t}\left(c_{1} w_{4 n}+g_{1} w_{2 n}\right) d \tau\right| \leqslant 0.16$.

We fix a point $x_{0}$ and consider the set $\Omega_{x_{0}}=\left\{x \mid x_{0}-0.16 \leqslant x \leqslant x_{0}+0.16\right\}$. Let us prove the equicontinuity of functions $\omega_{1}^{n}, \omega_{2}^{n}$ w.r.t. $x \in \Omega_{x_{0}}$, which implies the equicontinuity of functions $\omega_{1}^{n}, \omega_{2}^{n}$ w.r.t. $x$ in the chosen arbitrary point $x_{0}$, i.e., on $\mathbb{R}$. The equicontinuity of functions $\omega_{1}^{n}$, $\omega_{2}^{n}$ w.r.t. $x$ is employed in the proof of convergence for successive approximations $\omega_{j}^{n}, j=\overline{1,4}$.

We take $x_{1}, x_{2} \in \Omega_{x_{0}}$. Let us prove the inequalities

$$
\begin{aligned}
& \left|\eta_{1 n}\left(s, t, x_{1}\right)-\eta_{1 n}\left(s, t, x_{2}\right)\right| \leqslant\left|x_{1}-x_{2}\right|, \\
& \left|\eta_{2 n}\left(s, t, x_{1}\right)-\eta_{2 n}\left(s, t, x_{2}\right)\right| \leqslant\left|x_{1}-x_{2}\right|,
\end{aligned}
$$

where

$$
\begin{aligned}
& \eta_{1 n}(s, t, x)=x-\int_{s}^{t}\left(a_{1} w_{1 n}(\tau, t, x)+b_{1} w_{3 n}(\tau, t, x)\right) d \tau, \\
& \eta_{2 n}(s, t, x)=x-\int_{s}^{t}\left(c_{1} w_{4 n}(\tau, t, x)+g_{1} w_{2 n}(\tau, t, x)\right) d \tau .
\end{aligned}
$$

Differentiating successive approximations $(29)-(32)$ w.r.t. $x$, we obtain

$$
\begin{aligned}
w_{1 n x}= & \varphi_{1}^{\prime}\left(x-\int_{0}^{t}\left(a_{1} w_{1}+b_{1} w_{3}\right) d \tau\right) \cdot\left(1-\int_{0}^{t}\left(a_{1} w_{1 n x}+b_{1} w_{3 n x}\right) d \tau\right) \\
& +\int_{0}^{s}\left(a_{2} w_{1 n x}+b_{2} w_{3 n x}\right) d \tau
\end{aligned}
$$




$$
\begin{aligned}
& w_{2 n x}=\varphi_{2}^{\prime}\left(x-\int_{0}^{t}\left(c_{1} w_{4 n}+g_{1} w_{2 n}\right) d \tau\right) \cdot\left(1-\int_{0}^{t}\left(c_{1} w_{4 n x}+g_{1} w_{2 n x}\right) d \tau\right)+\int_{0}^{s} g_{2} w_{2 n x} d \tau, \\
& w_{3 n x}=w_{2(n-1) x} \cdot\left(1-\int_{s}^{t}\left(a_{1} w_{1 n x}+b_{1} w_{3 n x}\right) d \tau\right), \\
& w_{4 n x}=w_{1(n-1) x} \cdot\left(1-\int_{s}^{t}\left(c_{1} w_{4 n x}+g_{1} w_{2 n x}\right) d \tau\right) .
\end{aligned}
$$

System (43)-(46) is equivalent to

$$
\begin{aligned}
w_{1 n x}= & \varphi_{1}^{\prime}\left(x-\int_{0}^{t}\left(a_{1} w_{1}+b_{1} w_{3}\right) d \tau\right) \cdot\left(1-\int_{0}^{t}\left(a_{1} w_{1 n x}+b_{1} w_{3 n x}\right) d \tau\right) \exp \left(a_{2} s\right) \\
& +\int_{0}^{s} b_{2} w_{3 n x} \exp \left(a_{2}(s-\tau)\right) d \tau \\
w_{2 n x}= & \varphi_{2}^{\prime}\left(x-\int_{0}^{t}\left(c_{1} w_{4 n}+g_{1} w_{2 n}\right) d \tau\right) \cdot\left(1-\int_{0}^{t}\left(c_{1} w_{4 n x}+g_{1} w_{2 n x}\right) d \tau\right) \exp \left(g_{2} s\right), \\
w_{3 n x}= & w_{2(n-1) x} \cdot\left(1-\int_{s}^{t}\left(a_{1} w_{1 n x}+b_{1} w_{3 n x}\right) d \tau\right), \\
w_{4 n x}= & w_{1(n-1) x} \cdot\left(1-\int_{s}^{t}\left(c_{1} w_{4 n x}+g_{1} w_{2 n x}\right) d \tau\right) .
\end{aligned}
$$

We suppose that

$$
w_{1(n-1) x} \geqslant 0, \quad w_{2(n-1) x} \geqslant 0 .
$$

Under conditions (28), by means of the properties of integrals, absolute values and supremum of functions we prove that for each $n \in \mathbb{N}$ the inequalities

$$
1-\int_{s}^{t}\left(a_{1} w_{1 n x}+b_{1} w_{3 n x}\right) d \tau>0, \quad 1-\int_{s}^{t}\left(c_{1} w_{4 n x}+g_{1} w_{2 n x}\right) d \tau>0
$$

hold true on $\Gamma_{T_{2}}$. It follows from (49)-(52) that $w_{3 n x} \geqslant 0, w_{4 n x} \geqslant 0$.

Under conditions (28) by (47), 448) and inequalities (52) we get

$$
w_{1 n x} \geqslant 0, w_{2 n x} \geqslant 0 .
$$

Since $w_{1 n x} \geqslant 0, w_{2 n x} \geqslant 0, w_{3 n x} \geqslant 0, w_{4 n x} \geqslant 0$, we have

$$
1-\int_{s}^{t}\left(a_{1} w_{1 n x}+b_{1} w_{3 n x}\right) d \tau \leqslant 1, \quad 1-\int_{s}^{t}\left(c_{1} w_{4 n x}+g_{1} w_{2 n x}\right) d \tau \leqslant 1 .
$$

By inequalities (52), (53) and finite increment theorem we arrive at inequalities (41), (42).

Under conditions (28) by (37), (39) and the properties of integrals, absolute values and supremum of functions we get the inequalities $\left|\omega_{1}^{n}\left(s, t, x_{1}\right)-\omega_{1}^{n}\left(s, t, x_{2}\right)\right|<\Phi_{1 n}+\left(C_{\varphi} l t+l t\right)\left(\left|\omega_{1}^{n}\left(s, t, x_{1}\right)-\omega_{1}^{n}\left(s, t, x_{2}\right)\right|\right.$ 


$$
\begin{aligned}
& \left.+\left|\omega_{3}^{n}\left(s, t, x_{1}\right)-\omega_{3}^{n}\left(s, t, x_{2}\right)\right|\right) \\
\left|\omega_{3}^{n}\left(s, t, x_{1}\right)-\omega_{3}^{n}\left(s, t, x_{2}\right)\right|< & \Phi_{2 n}+\left|\omega_{2}^{n-1}\left(s, s, \eta_{1 n}\left(s, t, x_{1}\right)\right)-\omega_{2}^{n-1}\left(s, s, \eta_{1 n}\left(s, t, x_{2}\right)\right)\right| \\
& +4 C_{\varphi} l t\left(\left|\omega_{1}^{n}\left(s, t, x_{1}\right)-\omega_{1}^{n}\left(s, t, x_{2}\right)\right|+\left|\omega_{3}^{n}\left(s, t, x_{1}\right)-\omega_{3}^{n}\left(s, t, x_{2}\right)\right|\right),
\end{aligned}
$$

where

$$
\begin{aligned}
\Phi_{1 n}= & \mid\left(\varphi_{1}^{\prime \prime}\left(\eta_{1 n}\left(0, t, x_{1}\right)\right)-\varphi_{1}^{\prime \prime}\left(\eta_{1 n}\left(0, t, x_{2}\right)\right)\right) \eta_{1 n x}^{2}\left(s, t, x_{1}\right) \\
& +\varphi_{1}^{\prime \prime}\left(\eta_{1 n}\left(0, t, x_{2}\right)\right)\left[\eta_{1 n x}^{2}\left(0, t, x_{1}\right)-\eta_{1 n x}^{2}\left(0, t, x_{2}\right)\right] \\
& -\left(\varphi_{1}^{\prime}\left(\eta_{1 n}\left(0, t, x_{1}\right)\right)-\varphi_{1}^{\prime}\left(\eta_{1 n}\left(0, t, x_{2}\right)\right)\right) \cdot \int_{0}^{t}\left(a \omega_{1}^{n}\left(\tau, t, x_{1}\right)+b \omega_{3}^{n}\left(\tau, t, x_{1}\right)\right) d \tau \mid \\
\Phi_{2 n}= & \mid \omega_{2}^{n-1}\left(s, s, \eta_{1 n}\left(s, t, x_{2}\right)\right) \cdot\left[\eta_{1 n x}^{2}\left(s, t, x_{1}\right)-\eta_{1 n x}^{2}\left(s, t, x_{2}\right)\right] \\
& -\int_{s}^{t}\left(a \omega_{1}^{n}\left(\tau, t, x_{1}\right)+b \omega_{3}^{n}\left(\tau, t, x_{1}\right)\right) d \tau \\
& \cdot\left[w_{2(n-1) x}\left(s, s, \eta_{1 n}\left(s, t, x_{1}\right)\right)-w_{2(n-1) x}\left(s, s, \eta_{1 n}\left(s, t, x_{2}\right)\right)\right] \mid \\
\eta_{1 n}(s, & t, x)=x-\int_{s}^{t}\left(a w_{1 n}(\tau, t, x)+b w_{3 n}(\tau, t, x)\right) d \tau .
\end{aligned}
$$

Thus, on $\Gamma_{T_{2}}$, where $T_{2}=\min \left(\frac{1}{25 C_{\varphi} l}, \frac{1}{10 l}\right)$, the inequalities

$$
\begin{aligned}
\left|\omega_{1}^{n}\left(s, t, x_{1}\right)-\omega_{1}^{n}\left(s, t, x_{2}\right)\right|< & \Phi_{1 n}+0.14\left(\left|\omega_{1}^{n}\left(s, t, x_{1}\right)-\omega_{1}^{n}\left(s, t, x_{2}\right)\right|\right. \\
& \left.+\left|\omega_{3}^{n}\left(s, t, x_{1}\right)-\omega_{3}^{n}\left(s, t, x_{2}\right)\right|\right) \\
\left|\omega_{3}^{n}\left(s, t, x_{1}\right)-\omega_{3}^{n}\left(s, t, x_{2}\right)\right|< & \Phi_{2 n}+\left|\omega_{2}^{n-1}\left(s, s, \eta_{1 n}\left(s, t, x_{1}\right)\right)-\omega_{2}^{n-1}\left(s, s, \eta_{1 n}\left(s, t, x_{2}\right)\right)\right| \\
& +0.16\left(\left|\omega_{1}^{n}\left(s, t, x_{1}\right)-\omega_{1}^{n}\left(s, t, x_{2}\right)\right|+\left|\omega_{3}^{n}\left(s, t, x_{1}\right)-\omega_{3}^{n}\left(s, t, x_{2}\right)\right|\right)
\end{aligned}
$$

hold true.

Employing the uniform continuity and equicontinuity as well as the boundedness of all the functions in $\Phi_{1 n}, \Phi_{2 n}$, for each arbitrarily small number $\varepsilon$ we can choose $\delta>0$ such that $\Phi_{1 n}<0.5 \varepsilon, \Phi_{2 n}<0.5 \varepsilon$ as $\left|x_{1}-x_{2}\right|<\delta$ for each $n$.

Suppose that $\left|\omega_{2}^{(n-1)}\left(s, t, x_{1}\right)-\omega_{2}^{(n-1)}\left(s, t, x_{2}\right)\right|<\varepsilon$ as $\left|x_{1}-x_{2}\right|<\delta$. Then

$$
\begin{aligned}
\left|\omega_{1}^{n}\left(s, t, x_{1}\right)-\omega_{1}^{n}\left(s, t, x_{2}\right)\right|< & 0.5 \varepsilon+0.14\left(\left|\omega_{1}^{n}\left(s, t, x_{1}\right)-\omega_{1}^{n}\left(s, t, x_{2}\right)\right|\right. \\
& \left.+\left|\omega_{3}^{n}\left(s, t, x_{1}\right)-\omega_{3}^{n}\left(s, t, x_{2}\right)\right|\right), \\
\left|\omega_{1}^{n}\left(s, t, x_{1}\right)-\omega_{1}^{n}\left(s, t, x_{2}\right)\right|+ & \left|\omega_{3}^{n}\left(s, t, x_{1}\right)-\omega_{3}^{n}\left(s, t, x_{2}\right)\right|<\frac{20}{7} \varepsilon .
\end{aligned}
$$

Therefore, $\left|\omega_{1}^{n}\left(s, t, x_{1}\right)-\omega_{1}^{n}\left(s, t, x_{2}\right)\right|<\varepsilon$ as $\left|x_{1}-x_{2}\right|<\delta$. In the same way we obtain $\left|\omega_{2}^{n}\left(s, t, x_{1}\right)-\omega_{2}^{n}\left(s, t, x_{2}\right)\right|<\varepsilon$ as $\left|x_{1}-x_{2}\right|<\delta$. Thus, sequences $\left\{\omega_{i}^{n}(s, t, x)\right\}, i=1,2$, are equicontinuous w.r.t. $x \in \Omega_{x_{0}}$.

We consider the system of equations

$$
\tilde{\omega}_{1}^{n}=-\varphi_{1}^{\prime}\left(\eta_{1}(0, t, x)\right) \int_{0}^{t}\left(a_{1} \tilde{\omega}_{1}^{n}+b_{1} \tilde{\omega}_{3}^{n}\right) d \tau+\int_{0}^{s}\left(a_{2} \tilde{\omega}_{1}^{n}+b_{2} \tilde{\omega}_{3}^{n}\right) d \tau
$$




$$
\begin{gathered}
\quad+\varphi_{1}^{\prime \prime} \cdot\left(1-\int_{0}^{t}\left(a_{1} w_{1 x}+b_{1} w_{3 x}\right) d \tau\right)^{2}, \\
\tilde{\omega}_{2}^{n}=-\varphi_{2}^{\prime}\left(\eta_{2}(0, t, x)\right) \int_{0}^{t}\left(c_{1} \tilde{\omega}_{4}^{n}+g_{1} \tilde{\omega}_{2}^{n}\right) d \tau+\int_{0}^{s} g_{2} \tilde{\omega}_{2}^{n} d \tau+\varphi_{2}^{\prime \prime} \cdot\left(1-\int_{0}^{t}\left(c_{1} w_{4 x}+g_{1} w_{2 x}\right) d \tau\right)^{2}, \\
\tilde{\omega}_{3}^{n}=\tilde{\omega}_{2}^{n-1} \cdot\left(1-\int_{s}^{t}\left(a_{1} w_{1 x}+b_{1} w_{3 x}\right) d \tau\right)^{2}-w_{2 x}\left(s, s, \eta_{1}(s, t, x)\right) \int_{s}^{t}\left(a_{1} \tilde{\omega}_{1}^{n}+b_{1} \tilde{\omega}_{3}^{n}\right) d \tau, \\
\tilde{\omega}_{4}^{n}=\tilde{\omega}_{1}^{n-1} \cdot\left(1-\int_{s}^{t}\left(c_{1} w_{4 x}+g_{1} w_{2 x}\right) d \tau\right)^{2}-w_{1 x}\left(s, s, \eta_{2}(s, t, x)\right) \int_{s}\left(c_{1} \tilde{\omega}_{4}^{n}+g_{1} \tilde{\omega}_{2}^{n}\right) d \tau .
\end{gathered}
$$

Under condition (28), we obtain that $\tilde{\omega}_{j}^{n} \rightarrow \tilde{\omega}_{j}, j=\overline{1,4}$, on $\Gamma_{T_{2}}$ and the estimates

$$
\left\|\tilde{\omega}_{1}\right\| \leqslant 2 C_{\varphi}, \quad\left\|\tilde{\omega}_{2}\right\| \leqslant 2 C_{\varphi}, \quad\left\|\tilde{\omega}_{3}\right\| \leqslant 3 C_{\varphi}, \quad\left\|\tilde{\omega}_{4}\right\| \leqslant 3 C_{\varphi}
$$

hold true.

Inequality

$$
\left\|\omega_{1}^{N+k}-\tilde{\omega}_{1}\right\|+\left\|\omega_{2}^{N+k}-\tilde{\omega}_{2}\right\| \leqslant\left(\frac{1}{3}\right)^{k}\left(\left\|\omega_{1}^{N}-\tilde{\omega}_{1}\right\|+\left\|\omega_{2}^{N}-\tilde{\omega}_{2}\right\|\right)+4 \varepsilon
$$

yields that $\omega_{1}^{N+k} \rightarrow \tilde{\omega}_{1}, \omega_{2}^{N+k} \rightarrow \tilde{\omega}_{2}$ as $N \rightarrow \infty, k \rightarrow \infty$. We also get that $\omega_{3}^{n} \rightarrow \tilde{\omega}_{3}$ as $n \rightarrow \infty$, $\omega_{4}^{n} \rightarrow \tilde{\omega}_{4}$ as $n \rightarrow \infty$.

We obtain that $w_{j n x x} \rightarrow w_{j x x}=\tilde{\omega}_{j}$, where the functions $\frac{\partial^{2} w_{j}}{\partial x^{2}}, j=\overline{1,4}$, are continuous and bounded on $\Gamma_{T_{2}}$ under conditions (28). Thus, there exist continuous and bounded derivatives $\frac{\partial^{2} w_{j}}{\partial x t}, j=\overline{1,4}$ on $\Gamma_{T_{2}}$ under condition $(28)$.

\section{EXISTENCE OF NONLOCAL SOLUTION}

Theorem 2. Suppose that $\varphi_{i} \in \bar{C}^{2}(\mathbb{R}), i=1,2$, and conditions $(28)$ hold. Then for each $T>0$ Cauchy problem (4), (2) has the unique solution $u(t, x), v(t, x) \in \bar{C}^{1,2,2}\left(\Omega_{T}\right)$ determined by the system of integral equations (24)-27).

Proof. We differentiate the system of equations (4) w.r.t. $x$ and we denote $p(t, x)=u_{x}(t, x)$, $q(t, x)=v_{x}(t, x)$. It leads to the system of equations

$$
\left\{\begin{array}{l}
\partial_{t} p+\left(a_{1} u(t, x)+b_{1} v(t, x)\right) \partial_{x} p=-a_{1} p^{2}-b_{1} p q+a_{2} p+b_{2} q, \\
\partial_{t} q+\left(c_{1} u(t, x)+g_{1} v(t, x)\right) \partial_{x} q=-g_{1} q^{2}-c_{1} p q+g_{2} q, \\
p(0, x)=\varphi_{1}^{\prime}(x), \quad q(0, x)=\varphi_{2}^{\prime}(x) .
\end{array}\right.
$$

To system of equations (18)-23), we add extra two equations

$$
\left\{\begin{array}{l}
\frac{d \gamma_{1}(s, t, x)}{d s}=-a_{1} \gamma_{1}^{2}-b_{1} \gamma_{1} \gamma_{2}\left(s, s, \eta_{1}\right)+a_{2} \gamma_{1}+b_{2} \gamma_{2}\left(s, s, \eta_{1}\right), \\
\frac{d \gamma_{2}(s, t, x)}{d s}=-g_{1} \gamma_{2}^{2}-c_{1} \gamma_{1}\left(s, s, \eta_{2}\right) \gamma_{2}+g_{2} \gamma_{2} .
\end{array}\right.
$$

subject to the conditions $\gamma_{1}(0, t, x)=\varphi_{1}^{\prime}\left(\eta_{1}\right), \gamma_{2}(0, t, x)=\varphi_{2}^{\prime}\left(\eta_{2}\right)$. 
We rewrite system of equations (55) as

$$
\left\{\begin{array}{l}
\gamma_{1}(s, t, x)=\varphi_{1}^{\prime}\left(\eta_{1}\right)+\int_{0}^{s}\left[-a_{1} \gamma_{1}^{2}+\left(b_{2}-b_{1} \gamma_{1}\right) \gamma_{2}\left(\tau, \tau, \eta_{1}\right)+a_{2} \gamma_{1}\right] d \tau \\
\gamma_{2}(s, t, x)=\varphi_{2}^{\prime}\left(\eta_{2}\right)+\int_{0}^{s}\left[-g_{1} \gamma_{2}^{2}-c_{1} \gamma_{1}\left(\tau, \tau, \eta_{2}\right) \gamma_{2}+g_{2} \gamma_{2}\right] d \tau .
\end{array}\right.
$$

Under conditions (28), the existence of continuous solution to system (56) on $\Gamma_{T_{2}}$, where $T_{2}=\min \left(\frac{1}{25 C_{\varphi} l}, \frac{1}{10 l}\right)$, is made the successive approximations method. We introduce successive approximations:

$$
\left\{\begin{array}{l}
\gamma_{1}^{n+1}=\varphi_{1}^{\prime}\left(\eta_{1}\right)+\int_{0}^{s}\left[-a_{1}\left(\gamma_{1}^{n}\right)^{2}+\left(b_{2}-b_{1} \gamma_{1}^{n}\right) \gamma_{2}^{n}\left(\tau, \tau, \eta_{1}\right)+a_{2} \gamma_{1}^{n}\right] d \tau \\
\gamma_{2}^{n+1}=\varphi_{2}^{\prime}\left(\eta_{2}\right)+\int_{0}^{s}\left[-g_{1}\left(\gamma_{2}^{n}\right)^{2}-c_{1} \gamma_{1}^{n}\left(\tau, \tau, \eta_{2}\right) \gamma_{2}^{n}+g_{2} \gamma_{2}^{n}\right] d \tau
\end{array}\right.
$$

and $\gamma_{1}^{0}=\varphi_{1}^{\prime}\left(\eta_{1}\right), \gamma_{2}^{0}=\varphi_{2}^{\prime}\left(\eta_{2}\right)$.

Under conditions (28), the estimates $\left|\gamma_{i}^{n+1}\right| \leqslant 2 C_{\varphi},\left|\eta_{i x}\right| \leqslant 1,\left|\gamma_{i x}^{n+1}\right| \leqslant 5 C_{\varphi}, i=1,2$, hold true on $\Gamma_{T_{2}}$. Let us prove the convergence of successive approximations on $\Gamma_{T_{2}}$. We consider the inequalities:

$$
\begin{aligned}
\left|\gamma_{1}^{n+1}-\gamma_{1}^{n}\right| \leqslant & \left|\int_{0}^{s}\left[a_{1}\left(\left(\gamma_{1}^{n}\right)^{2}-\left(\gamma_{1}^{n-1}\right)^{2}\right)+b_{1}\left(\gamma_{1}^{n} \gamma_{2}^{n}-\gamma_{1}^{n-1} \gamma_{2}^{n-1}\right)\right] d \tau\right| \\
& +\left|\int_{0}^{s}\left[\left(\gamma_{1}^{n}-\gamma_{1}^{n-1}\right) a_{2}+\left(\gamma_{2}^{n}-\gamma_{2}^{n-1}\right) b_{2}\right] d \tau\right| \\
\leqslant & l t\left(\left\|\gamma_{1}^{n}+\gamma_{1}^{n-1}\right\| \cdot\left\|\gamma_{1}^{n}-\gamma_{1}^{n-1}\right\|+\left\|\gamma_{1}^{n}-\gamma_{1}^{n-1}\right\| \cdot\left\|\gamma_{2}^{n}\right\|+\left\|\gamma_{2}^{n}-\gamma_{2}^{n-1}\right\| \cdot\left\|\gamma_{1}^{n-1}\right\|\right) \\
& +l t\left(\left\|\gamma_{2}^{n}-\gamma_{2}^{n-1}\right\|+\left\|\gamma_{1}^{n}-\gamma_{1}^{n-1}\right\|\right) .
\end{aligned}
$$

Taking into considerations the estimates $\left|\gamma_{i}^{n+1}\right| \leqslant 2 C_{\varphi}, i=1,2$, we obtain

$$
\left\|\gamma_{1}^{n+1}-\gamma_{1}^{n}\right\| \leqslant\left(6 l t C_{\varphi}+l t\right)\left\|\gamma_{1}^{n}-\gamma_{1}^{n-1}\right\|+\left(2 l t C_{\varphi}+l t\right)\left\|\gamma_{2}^{n}-\gamma_{2}^{n-1}\right\| .
$$

In the same way we arrive at the inequality

$$
\left\|\gamma_{2}^{n+1}-\gamma_{2}^{n}\right\| \leqslant\left(6 l t C_{\varphi}+l t\right)\left\|\gamma_{2}^{n}-\gamma_{2}^{n-1}\right\|+2 l t C_{\varphi}\left\|\gamma_{1}^{n}-\gamma_{1}^{n-1}\right\| .
$$

We sum up the latter inequalities to obtain

$$
\left\|\gamma_{1}^{n+1}-\gamma_{1}^{n}\right\|+\left\|\gamma_{2}^{n+1}-\gamma_{2}^{n}\right\| \leqslant\left(8 l t C_{\varphi}+2 l t\right)\left(\left\|\gamma_{2}^{n}-\gamma_{2}^{n-1}\right\|+\left\|\gamma_{1}^{n}-\gamma_{1}^{n-1}\right\|\right) .
$$

We see that for $0 \leqslant t \leqslant T_{2}$, where $T_{2}=\min \left(\frac{1}{25 C_{\varphi} l}, \frac{1}{10 l}\right)$, the inequality

$$
\left\|\gamma_{1}^{n+1}-\gamma_{1}^{n}\right\|+\left\|\gamma_{2}^{n+1}-\gamma_{2}^{n}\right\| \leqslant 0.52\left(\left\|\gamma_{1}^{n}-\gamma_{1}^{n-1}\right\|+\left\|\gamma_{2}^{n}-\gamma_{2}^{n-1}\right\|\right)
$$

holds true. Thus, we conclude that successive approximations $\gamma_{i}^{n}, i=1,2$, converge to a continuous solution of system (56) in $\Gamma_{T_{2}}$ under conditions (28). This solutions satisfies the estimates $\left|\gamma_{i}\right| \leqslant 2 C_{\varphi}, i=1,2$. 
We consider the system of equations

$$
\left\{\begin{array}{l}
\omega_{21}=\varphi_{1}^{\prime \prime}\left(\eta_{1}\right) \eta_{1 x}+\int_{0}^{s}\left[\left(a_{2}-2 a_{1} \gamma_{1}-b_{1} \gamma_{2}\left(\tau, \tau, \eta_{1}\right)\right) \omega_{21}+\left(b_{2}-b_{1} \gamma_{1}\right) \omega_{22}\left(\tau, \tau, \eta_{1}\right) \eta_{1 x}\right] d \tau \\
\omega_{22}=\varphi_{2}^{\prime \prime}\left(\eta_{2}\right) \eta_{2 x}+\int_{0}^{s}\left[\left(g_{2}-2 g_{1} \gamma_{2}-c_{1} \gamma_{1}\left(\tau, \tau, \eta_{2}\right)\right) \omega_{22}-c_{1} \omega_{21}\left(\tau, \tau, \eta_{2}\right) \gamma_{2} \eta_{2 x}\right] d \tau
\end{array}\right.
$$

The proof of existence of continuous solution to system (58) is made by the successive approximations method

$$
\left\{\begin{aligned}
\omega_{21}^{n+1}= & \varphi_{1}^{\prime \prime}\left(\eta_{1}\right) \eta_{1 x} \\
& +\int_{0}^{s}\left[\left(a_{2}-2 a_{1} \gamma_{1}-b_{1} \gamma_{2}\left(\tau, \tau, \eta_{1}\right)\right) \omega_{21}^{n}+\left(b_{2}-b_{1} \gamma_{1}\right) \omega_{22}^{n}\left(\tau, \tau, \eta_{1}\right) \eta_{1 x}\right] d \tau, \\
\omega_{22}^{n+1}= & \varphi_{2}^{\prime \prime}\left(\eta_{2}\right) \eta_{2 x}+\int_{0}^{s}\left[\left(g_{2}-2 g_{1} \gamma_{2}-c_{1} \gamma_{1}\left(\tau, \tau, \eta_{2}\right)\right) \omega_{22}^{n}-c_{1} \omega_{21}^{n}\left(\tau, \tau, \eta_{2}\right) \gamma_{2} \eta_{2 x}\right] d \tau .
\end{aligned}\right.
$$

Under conditions (28), the estimates $\| \omega_{2 i}^{n+1} \mid \leqslant 5 C_{\varphi}, i=1,2$, hold true on $\Gamma_{T_{2}}$.

We consider the inequalities

$$
\begin{aligned}
\left|\omega_{21}^{n+1}-\omega_{21}^{n}\right| \leqslant & l \int_{0}^{s}\left(1+2\left|\gamma_{1}\right|+\left|\gamma_{2}\right|\right)\left|\omega_{21}^{n}-\omega_{21}^{n-1}\right| d \tau \\
& +l \int_{0}^{s}\left(1+\left|\gamma_{1}\right|\right) \cdot\left|\omega_{22}^{n}\left(\tau, \tau, \eta_{1}\right)-\omega_{22}^{n-1}\left(\tau, \tau, \eta_{1}\right)\right| d \tau .
\end{aligned}
$$

Taking into consideration the estimates $\left|\gamma_{i}\right| \leqslant 2 C_{\varphi}, i=1,2$, we obtain

$$
\left\|\omega_{21}^{n+1}-\omega_{21}^{n}\right\| \leqslant\left(6 l t C_{\varphi}+l t\right)\left\|\omega_{21}^{n}-\omega_{21}^{n-1}\right\|+\left(2 l t C_{\varphi}+l t\right)\left\|\omega_{22}^{n}-\omega_{22}^{n-1}\right\| .
$$

In the same way we arrive at the inequality:

$$
\left\|\omega_{22}^{n+1}-\omega_{22}^{n}\right\| \leqslant\left(6 l t C_{\varphi}+l t\right)\left\|\omega_{22}^{n}-\omega_{22}^{n-1}\right\|+\left(2 l t C_{\varphi}+l t\right)\left\|\omega_{21}^{n}-\omega_{21}^{n-1}\right\| .
$$

We sum up the latter inequalities to obtain

$$
\left\|\omega_{21}^{n+1}-\omega_{21}^{n}\right\|+\left\|\omega_{22}^{n+1}-\omega_{22}^{n}\right\| \leqslant\left(8 l t C_{\varphi}+2 l t\right)\left(\left\|\omega_{21}^{n}-\omega_{21}^{n-1}\right\|+\left\|\omega_{22}^{n}-\omega_{22}^{n-1}\right\|\right) .
$$

We see that for each $0 \leqslant t \leqslant T_{2}$, where $T_{2}=\min \left(\frac{1}{25 C_{\varphi} l}, \frac{1}{10 l}\right)$, the inequality

$$
\left\|\omega_{21}^{n+1}-\omega_{21}^{n}\right\|+\left\|\omega_{22}^{n+1}-\omega_{22}^{n}\right\| \leqslant 0.52\left(\left\|\omega_{21}^{n}-\omega_{21}^{n-1}\right\|+\left\|\omega_{22}^{n}-\omega_{22}^{n-1}\right\|\right)
$$

holds true.

Therefore, successive approximations $\left\{\omega_{2 i}^{n}\right\}, i=1,2$, converge to a continuous solution of system (58) on $\Gamma_{T_{2}}$ under conditions (28).

Inequality

$$
\left\|\gamma_{1 x}^{N+p}-\omega_{21}\right\|+\left\|\gamma_{2 x}^{N+p}-\omega_{22}\right\| \leqslant(0.52)^{p}\left(\left\|\gamma_{1 x}^{N}-\omega_{21}\right\|+\left\|\gamma_{2 x}^{N}-\omega_{22}\right\|\right)+3 \varepsilon
$$

follows that $\left\|\gamma_{1 x}^{N+p}-\omega_{21}\right\|+\left\|\gamma_{2 x}^{N+p}-\omega_{22}\right\| \rightarrow 0$ as $N \rightarrow \infty, p \rightarrow \infty$. Thus, $\lim _{n \rightarrow \infty} \gamma_{i x}{ }^{n}=\omega_{2 i}$, $i=1,2$. Therefore, there exists a continuous derivative w.r.t. $x$ of the solution to system (56), $\gamma_{i x}=\frac{\partial \gamma_{i}}{\partial x}=\omega_{2 i}$, and the estimates $\left\|\gamma_{i x}\right\| \leqslant 5 C_{\varphi}, i=1,2$, hold true. In the same way as in paper [3], we prove the existence of continuous derivative w.r.t. $t$ of the solution to system 
(56). Since there exists a differentiable solution to problem (56), then $\gamma_{1}(t, t, x)=p(t, x)=\partial_{x} u$, $\gamma_{2}(t, t, x)=q(t, x)=\partial_{x} v$.

In the same way as in [3] we establish that successive approximations (57) converge to the continuous solution to system (56) possessing continuous derivatives w.r.t. $t$ and $x$.

Therefore, $\gamma_{1}(t, t, x)=p(t, x)=\partial_{x} u, \gamma_{2}(t, t, x)=q(t, x)=\partial_{x} v$.

It follows from (13)-(14) that

$$
w_{1}(s, t, x)=\varphi_{1}\left(\eta_{1}\right) \exp \left(a_{2} s\right)+\int_{0}^{s} b_{2} w_{3} \exp \left(a_{2}(s-\tau)\right) d \tau, \quad w_{2}(s, t, x)=\varphi_{2}\left(\eta_{2}\right) \exp \left(g_{2} s\right) .
$$

We obtain that

$$
\left\|w_{2}\right\| \leqslant C_{\varphi} \exp \left(\left|g_{2}\right| T\right), \quad\left\|w_{1}\right\| \leqslant C_{\varphi} \exp \left(\left|a_{2}\right| T\right)\left(1+T b_{2} \exp \left(\left|g_{2}\right| T\right)\right) .
$$

Therefore, the estimates

$$
\|v\| \leqslant C_{\varphi} \exp \left(\left|g_{2}\right| T\right),\|u\| \leqslant C_{\varphi} \exp \left(\left|a_{2}\right| T\right)\left(1+T b_{2} \exp \left(\left|g_{2}\right| T\right)\right)
$$

hold true.

By (55) we get

$$
\left\{\begin{aligned}
\gamma_{1}(s, t, x)= & \varphi_{1}^{\prime}\left(\eta_{1}\right) \exp \left(-\int_{0}^{s}\left(a_{1} \gamma_{1}+b_{1} \gamma_{2}\left(\tau, \tau, \eta_{1}\right)-a_{2}\right) d \tau\right) \\
& +\int_{0}^{s} b_{2} \gamma_{2}\left(\tau, \tau, \eta_{1}\right) \exp \left(-\int_{\tau}^{s}\left(a_{1} \gamma_{1}+b_{1} \gamma_{2}\left(\nu, \nu, \eta_{1}\right)-a_{2}\right) d \nu\right) d \tau, \\
\gamma_{2}(s, t, x)= & \varphi_{2}^{\prime}\left(\eta_{2}\right) \exp \left(-\int_{0}^{s}\left(g_{1} \gamma_{2}+c_{1} \gamma_{1}\left(\tau, \tau, \eta_{2}\right)-g_{2}\right) d \tau\right) .
\end{aligned}\right.
$$

Under conditions (28) we obtain $\gamma_{i} \geqslant 0, i=1,2$, hence, $\left\|\gamma_{2}\right\| \leqslant C_{\varphi} \exp \left(\left|g_{2}\right| T\right),\left\|\gamma_{1}\right\| \leqslant$ $C_{\varphi} \exp \left(\left|a_{2}\right| T\right)\left(1+T b_{2} \exp \left(\left|g_{2}\right| T\right)\right)$. It implies the estimates

$$
\left\|\partial_{x} v\right\| \leqslant C_{\varphi} \exp \left(\left|g_{2}\right| T\right),\left\|\partial_{x} u\right\| \leqslant C_{\varphi} \exp \left(\left|a_{2}\right| T\right)\left(1+T b_{2} \exp \left(\left|g_{2}\right| T\right)\right) .
$$

In the same way as in the paper [3] we establish that for each $t$ and $x$ the estimates

$$
\begin{aligned}
& \left|\partial_{x^{2}}^{2} u\right| \leqslant E_{11} \cosh \left(t \sqrt{C_{12} C_{21}}\right)+E_{21} \sqrt{\frac{C_{12}}{C_{21}}} \sinh \left(t \sqrt{C_{12} C_{21}}\right), \\
& \left|\partial_{x^{2}}^{2} v\right| \leqslant E_{21} \cosh \left(t \sqrt{C_{12} C_{21}}\right)+E_{11} \sqrt{\frac{C_{21}}{C_{12}}} \sinh \left(t \sqrt{C_{12} C_{21}}\right),
\end{aligned}
$$

where $E_{11}, E_{21}, C_{12}, C_{21}$ are constants determined by initial data.

The obtained global estimates (60), (62)-(64) allow us to continue the solution on each given segment $[0, T]$. Taking $u\left(T_{0}, x\right), v\left(T_{0}, x\right)$ as the initial conditions, we continue the solution to some segment $\left[T_{0}, T_{1}\right]$, and then, taking $u\left(T_{1}, x\right), v\left(T_{1}, x\right)$ as the initial condition, we continue the solution to the segment $\left[T_{1}, T_{2}\right]$. The length of the resolvability interval does not decreases since it is determined by the quantities $\left\|\partial_{x} u\right\|,\left\|\partial_{x} v\right\|$ bounded by global estimates (62) valid on each resolvability interval. The second derivatives satisfy estimates (63), (64), where we take $T$ as $t$. As a result, in a finite number of steps, we can continue the solution to each given segment $[0, T]$.

The uniqueness of solution to Cauchy problem (4), (2) can be proven by applying estimates similar to those used in the proof of convergence of successive approximations. 
Exmaple. We consider the Cauchy problem for the system

$$
\left\{\begin{array}{l}
\partial_{t} u(t, x)+(u(t, x)+4 v(t, x)) \partial_{x} u(t, x)=-2 u(t, x)+3 v(t, x), \\
\partial_{t} v(t, x)+(7 u(t, x)+5 v(t, x)) \partial_{x} v(t, x)=-v(t, x),
\end{array}\right.
$$

where $u(t, x), v(t, x)$ are unknown functions, $(t, x) \in \Omega_{T}$ subject to the initial conditions:

$$
u(0, x)=\varphi_{1}(x)=\frac{\arctan x}{4}, \quad v(0, x)=\varphi_{2}(x)=-\frac{1}{5\left(e^{x}+1\right)} .
$$

Since $\varphi_{i} \in \bar{C}^{2}(\mathbb{R}), i=1,2, a_{1}=1>0, b_{1}=4>0, c_{1}=7>0, g_{1}=5>0, b_{2}=3>0$, $\varphi_{1}^{\prime}(x)=\frac{1}{4\left(1+x^{2}\right)}>0, \varphi_{2}^{\prime}(x)=\frac{e^{x}}{5\left(e^{x}+1\right)^{2}}>0$, by Theorem 2, Cauchy problem 65, 66 has the unique solution $u(t, x), v(t, x) \in \bar{C}^{1,2,2}\left(\Omega_{T}\right)$.

\section{BIBLIOGRAPHY}

1. B.L. Rozhdestvenskij, N.N. Yanenko. Systems of quasilinear equations and their applications to gas dynamics. Nauka, Moscow (1978). [Amer. Math. Soc., Providence, RI (1983).]

2. M.I. Imanaliev, P.S. Pankov, S.N. Alekseenko. Additional argument method // Vestnik KazNU. Ser. Matem. mech. inform. 1, 60-64 (2006). (in Russian).

3. S.N. Alekseenko, T.A. Shemyakina, M.V. Dontsova. Nonlocal solvability conditions for system of first order partial differential equations // Nauchno-tekhnicheskie vedomosti SPbGBU. Fiz.-Mat. Nauki. 3(177). 190-201 (2013). (in Russian).

4. M.V. Dontsova. Study of solvability for a system of first order partial differential equations with free terms // Proc. of International Youth Conference "Lomonosov-2014", (2014). (in Russian).

5. L.G. Loitsyanskii. Mechanics of Liquids and Gases. Nauka, Moscow (1950). [Int. Ser. Monog. Aeronautics Astronautics. 6. Pergamon Press, Oxford (1966).]

6. F.I. Frankl. Selected works on gas dynamics Nauka, Moscow (1973). (in Russian).

7. T.A. Shemyakina. Existence theorem for bounded solution to Cauchy problem for Frankl system of hyperbolic type // Nauchno-tekhnicheskie vedomosti SPbGBU. Fiz.-Mat. Nauki. 2(146), 130-131 (2012). (in Russian).

8. S.N. Alekseenko, M.V. Dontsova. Study of solvability for system of equations describing electrons distribution in electrical field of sprite // Matem. Vestn. Pedvuzov Univ. Volgo-Vyatskogo regiona. 14, 34-41 (2012). (in Russian).

9. S.N. Alekseenko, M.V. Dontsova. Local existence of bounded solution to system of equations describing electrons distribution in weakly ionized plasma in eletrical field of sprite // Matem. Vestn. Pedvuzov Univ. Volgo-Vyatskogo regiona. 15, 52-59 (2013).

10. M.V. Dontsova. Local solvability conditions of Cauchy problem for system of equations describing electrons distribution in weakly ionized plasma in eletrical field of sprite // Proc. of XVIIIth Nizhny Novgorod session of young scientist. Natural Math. Sci. 183-185 (2013).

11. M.I. Imanaliev, Yu.A. Ved'. First-order partial differential equation with an integral as a coefficient // Differ. Uravn. 25:3, 465-477 (1989). [Differ. Equat. 25:3, 325-335 (1989).]

12. M.I. Imanaliev, S.N. Alekseenko. On the theory of systems of nonlinear integro-partial differential equations of Whitham type // Dokl. Ross. Akad. Nauk. 325:6, 1111-1115 (1992). [Dokl. Math. 46:1, 169-173 (1993)].

13. M.I. Imanaliev, S.N. Alekseenko. On the existence of a smooth bounded solution for a system of two first-order nonlinear partial differential equations // Dokl. Ross. Akad. Nauk. 379:1, 16-21 (2001). [ Dokl. Math. 64:1, 10-15 (2001).]

Dontsova Marina Vladimirovna,

Minin Nizhny Novgorod State Pedagogical University,

ulyanova str., 1 ,

603950, Nizhny Novgorod, Russia

E-mail: dontsowa.marina2011@yandex.ru 